\title{
Quaderni
}

QUADERN I Communication, technologies, pouvoir

73 | Automne 2010

La métropole parisienne entre récits, paroles et échanges

\section{Entretien avec Pierre Mansat}

\section{Pierre Mansat}

\section{(2) OpenEdition \\ Journals}

Édition électronique

URL : http://journals.openedition.org/quaderni/453

DOI : 10.4000/quaderni.453

ISSN : 2105-2956

Éditeur

Les éditions de la Maison des sciences de l'Homme

Édition imprimée

Date de publication : 5 octobre 2010

Pagination : 89-96

Référence électronique

Pierre Mansat, «Entretien avec Pierre Mansat », Quaderni [En ligne], 73 | Automne 2010, mis en ligne le 05 octobre 2012, consulté le 19 avril 2019. URL : http://journals.openedition.org/quaderni/453 ; DOI :

10.4000/quaderni.453 


\section{$D$ ossier}

\section{entretien avec Q: comment pouver-vous ressumer aujour- d'hui le projet de Paris Métropole, sa dyna- Pierre Mansat mique, son inscription progressive dans l'agenda politique ?}

Il faut se souvenir que, jusqu'à ces deux ou trois adjoint au Maire de Paris, chargé de Paris Métropole et des relations avec les collectivités territoiriales d'île-de-France dernières années, le terme même de métropole était inconnu du langage politique. De ce point de vue, l'inscription à l'agenda politique de la question de la métropole parisienne a pu sembler soudaine. En réalité, l'apparition du projet de Paris Métropole est le fruit d'un long travail d'influence intellectuelle et politique que l'on peut faire remonter à l'élection de Bertrand Delanoë, en 2001. C'est à ce moment-là que s'engage politiquement le projet de renouveler les relations de Paris avec les collectivités territoriales d'îlede-France. Il a donc fallu d'abord inscrire cette nouvelle logique à l'agenda politique parisien, en faisant naître une conscience métropolitaine. Et très rapidement, ensuite, faire émerger des projets " d'intérêt commun », avec tous les niveaux de collectivités de l'agglomération, pour « déminer» l'histoire compliquée de Paris et ce qu'on appelait alors «sa » périphérie.

C'est autour de 2005-2006, que la dynamique parisienne devient une dynamique métropolitaine. La Conférence métropolitaine est alors lancée, comme un lieu informel où se rencontrent des élus de bonne volonté, disposés à plancher sans œillères ni présupposés sur ces questions. Alors que certains, du moins au début, voyaient dans la conférence métropolitaine un outil de dialogue entre Paris et ses voisins, d'autres - je pense à Daniel Guiraud, à Philippe Laurent, à Jacques Martin, notamment - ont rapidement affirmé qu'il fallait sans attendre réfléchir à une échelle plus 
large. Il ne s'agissait pas d'aider Paris à résoudre ses problèmes mais de résoudre les problèmes de la métropole: la question du logement, celle du développement économique, des déplacements, du rapport au savoir.

Le chef de l'État, rompant avec des décennies de désengagement de l'État en Île-de-France, s'est très vite exprimé en faveur d'une communauté urbaine sur le modèle de ce qui peut exister à Lyon, Toulouse... La désignation de Christian Blanc, comme secrétaire d'État chargé du Développement de la région capitale, mais surtout le lancement, avec le soutien de la ville de Paris, d'une consultation internationale, " le Grand Pari(s) de l'agglomération parisienne » ont donné à ce sujet une visibilité nationale.

C'est sur ce fond politique, que la conférence métropolitaine s'est transformée en Paris Métropole, en 2009. Nous avons fait le choix de retenir la forme d'un syndicat d'étude pour faciliter la coordination des politiques publiques, l'émergence de projets partagés et la consolidation d'une culture métropolitaine. Aujourd'hui, donc, le projet de Paris Métropole, scène politique d'un genre nouveau, atteint une certaine maturité.

\section{Q : Comment décrivez-vous le rôle politique que vous avez joué dans son émergence, son déploiement et son appropriation collective ?}

Le rôle politique que j'ai assumé a évidemment évolué à mesure que le projet métropolitain prenait corps, mais le mandat que m'a donné B. Delanoë a été constant sur un point : donner à Paris une place essentielle pour structurer cet élan. Il est impossible de nier que Paris ait à jouer un rôle moteur dans la construction de cette nouvelle scène politique. C'est Paris qui donne au projet sa raison d'être, mais qui est surtout le lien nécessaire, le trait d'union entre les territoires. C'est également Paris qui doit convaincre que l'enjeu n'est pas d'installer une nouvelle forme d'hégémonie, mais bien de réunir des forces dispersées et le plus souvent concurrentes pour une mise en cohérence des projets.

Initialement, ce rôle a pris la forme d'un travail patient de connaissance et de reconnaissance mutuelle, pour restaurer la confiance avec les élus franciliens. J'ai voulu avancer sur deux plans : la connaissance et l'action. L'effort de compréhension a été important, compréhension de ce territoire et de ses enjeux, mais aussi de la manière de "faire la ville ». Par ailleurs, pour inventer cette autre manière de faire de la politique dans le domaine urbain mais aussi culturel, économique, il fallait impulser une foule d'actes concrets, quotidiens.

Cette manière de faire était directement issue de mon expérience d'élu en charge du conseil de quartier périphérique dans le $\mathrm{XX}^{\mathrm{e}}$ arrondissement. C'est à ce moment-là que j'ai identifié ceux qui pouvaient constituer les défricheurs, mais aussi les « relais intellectuels » de cette nouvelle manière d'aborder l'action politique. En 1999, le réseau «Paris métropole ouverte» que je crée avec plusieurs personnalités qui s'étaient emparées de la question comme Yves Lion (il avait publié un manifeste décisif : «75021, appel pour une métropole nommée Paris») forme vraiment le laboratoire de cette action.

Arrivé à l'Hôtel de Ville, les services sur 
lesquels je devais m'appuyer avaient des moyens très limités. C'est pourquoi j'ai constitué très tôt un «comité des experts». L'idée était de réunir différents scientifiques pensant le sujet (géographes, urbanistes, architectes, économistes et sociologues) et de créer un lieu où des éléments d'analyse sur la place de Paris dans le système francilien se constituent.

Cet aller-retour entre réflexion et action est une sorte de spécificité de la démarche parisienne : les idées ont cheminé, marquées par des actes concrets. L'exemple récent d'Autolib' témoigne $\mathrm{du}$ chemin parcouru. Paris aurait pu lancer ce nouveau service à l'échelle strictement parisienne. En le faisant d'emblée sur un bassin plus large (Paris a élaboré ce projet avec 30 collectivités), le gain est évident, pour l'écologie et pour les usagers.

\section{Q : Selon vous, quelle place les médias ont pris dans la publicisation du projet ?}

Cette histoire s'est construite malgré l'indifférence des médias, sauf exception. Le champ médiatique a longtemps reproduit le profond émiettement politique de l'̂̂le-de-France ; Le Parisien, qui multiplie les éditions départementales mais a du mal à traiter l'espace métropolitain comme un ensemble, en est un bon exemple. Encore aujourd'hui, il est frappant de constater qu'il n'existe pas de vrai média métropolitain. L'analyse des échecs répétés ( 7 à Paris, Zurban, le supplément Paris Obs) ne me semble pas avoir été faite.

Le territoire métropolitain n'a pas ainsi de représentation médiatique, ou alors sous des formes limitées : la page « Grand Paris » de 20 minutes continue de constituer une exception. J'ai toujours considéré que cette absence de représentation collective était, en soi, un problème politique. Elle freine considérablement la construction d'une identité partagée. C'est pour cette raison que j'ai tenté d'accompagner tous les projets qui ont émergé à Paris pour créer un media métropolitain. Le dernier-né, Mégalopolis, bimestriel, diffusé depuis février, qui se donne pour but d'accompagner l'émergence du grand Paris au travers de sujets de société, de politique ou encore de sports et culture, aura peut-être plus de chance que ses prédécesseurs. Je ne connais pas à Paris d'équivalent de Place publique, la revue de débat sur les questions urbaines qui a accompagné l'émergence de la métropole de Nantes/ Saint-Nazaire.

Cette place modeste dans les médias s'est progressivement élargie à mesure que la conscience métropolitaine s'est enracinée. À partir de 2007, bien sûr, autour des résultats de la consultation internationale, aussi. On a pu alors s'appuyer sur la continuité de l'engagement parisien, sur le réseau constitué autour des quelques défricheurs du sujet, mais aussi de tous ceux qui ont, à un moment ou à un autre, tenté de faire naître ce média métropolitain.

Il faut souligner que quelques journalistes se sont intéressés très tôt à la question. Ils ont joué un rôle essentiel, que cela soit autour de Paris Obs (Guillaume Malaurie), ou de Libération. Mais, à l'exception du débat politique au sens étroit, ce sujet continue d'exister difficilement dans les pages des quotidiens, même pour un journal de référence comme Le Monde. 
Q : Quelle politique de communication avezvous mis en place pour accompagner votre action ? et, rétrospectivement, quelles ont été ses forces et ses faiblesses ?

Face à ce silence, et avec des moyens extrêmement limités, une politique très ciblée a été mise en place. On n'avait pas d'autre choix que d'occuper des espaces moins médiatiques, mais qui permettaient d'installer le sujet et ainsi de s'imposer dans le champ intellectuel, avant d'accéder à une plus grande visibilité médiatique. La politique de communication s'est donc appuyée sur l'organisation de séminaires, de colloques, relayés essentiellement au travers de publications et d'expositions.

Toute l'action de coopération autour de projets a été ainsi rythmée de moments de remise en perspectives, de réflexions partagées. En 2002, l'exposition L'archipel métropolitain, montée à l'Arsenal (et rapidement suivie d'un livre qui a réuni plus de 100 auteurs) a posé les bases de la problématique métropolitaine. Rétrospectivement, on peut dire que les grands thèmes dont on discute aujourd'huiy sont déjà posés.

Je crois que cette communication n'a été crédible que parce qu'elle n'a jamais oublié les projets très concrets. Au contraire, elle revisitait le « réel » des Parisiens mais pour constituer le point de départ d'un projet politique. Le travail que j'ai lancé autour du périphérique avec l'édition d'un collectif d'architectes, Tomato, est emblématique de cette approche. Pour la première fois, il s'agissait de regarder le périphérique non pas seulement comme un ouvrage de voirie ou comme générateur de nuisances, mais comme la « ville du périphérique ». Ce livre a marqué le début d'une réflexion très riche autour de ce symbole, poursuivie entre 2006 et 2008 par une étude d'insertion urbaine co-dirigée avec la Région et menée en concertation avec les villes riveraines.

Le programme de recherche conduit par Annie Fourcaut sur « L'histoire de Paris et de ses banlieues à l'époque contemporaine » est un autre jalon important. En termes de communication et sur le fond. Il a corrigé un cliché largement installé, en permettant de montrer que les rapports Paris / Banlieues ne pouvaient pas être réduits à l'histoire binaire de la domination unilatérale d'un centre omnipotent et prédateur, et de la relégation de périphéries uniformément misérables et servantes.

En plus de ces outils classiques, nous avons vite fait le pari d'utiliser les nouveaux supports de communication pour diffuser les résultats de ces travaux et faire connaître les premières réalisations. Le site internet de la Ville, www. paris.fr a constitué un relais intéressant. On l'a investi pour en faire le miroir de toute notre action. On y trouvait, dès 2002, à la fois le compte rendu des projets bilatéraux développés par Paris mais aussi un espace de «contributions et débats», qui est très vite devenu le lieu de diffusion des réflexions des experts, français et étrangers, que nous mobilisions. La création de mon blog (www.pierremansat.com) sous le nom de « Paris Métropole fédérée » m'a permis de disposer d'un support moins institutionnel et plus propice à une expression politique. J'en ai fait, au cours des années une véritable plateforme de la construction métropolitaine. 
Enfin, dès décembre 2001 nous avons lancé une lettre trimestrielle, Extramuros. Diffusée gratuitement jusqu'en 2008 à tous les élus et professionnels du secteur mais aussi à tout particulier qui en faisait la demande, elle rend compte de l'actualité de la coopération territoriale et du débat d'idées. Si la diffusion ne dépassera jamais les 7000 exemplaires, cet outil contribue à faire connaître le sujet.

En définitive, la communication sous différentes formes a toujours obéi à une politique de stimuli de la pensée métropolitaine. Dans le fond, je crois que cet axe politique n'est devenu visible que parce qu'il a contribué à rendre plus apparents des enjeux plus profonds. Tout l'effort a consisté à savoir changer de formats, de supports et à renouveler les acteurs, selon les moments politiques. Mon cycle de conférences mensuelles, les Mini-métropolitaines, vise aujourd'hui à convaincre les cadres de la Ville de Paris d'adopter un nouveau mode de réflexion.

On peut considérer que la combinaison de ces différents outils a permis, malgré leur modestie, l'émergence en à peine 10 ans, d'un sujet qui n'existait pas sur la scène politique. La principale faiblesse est de ne pas être parvenu à créer assez tôt un événement suffisamment marquant pour conserver le temps d'avance que nous avions.

\section{Q : Pensez vous que l'actuel débat public autour de la question de la gouvernance soit accessible aux citoyens ?}

Le débat tel qu'il est posé, il faut le reconnaître, est assez inaccessible. La question de la gouvernance a en effet été appropriée par un débat très expert.
La toile de fond est vraiment difficile à démêler pour les citoyens, qui ont du mal à se repérer dans l'enchevêtrement de structures, de compétences et financements : commune, intercommunalité, département, région...

Ce constat est assez décevant pour moi qui ai toujours pensé que le projet métropolitain avait comme premier objectif de répondre aux difficultés quotidiennes des habitants de la métropole. On avait là une occasion rare d'inventer les termes d'un débat citoyen avec une part de démocratie participative. Le risque, c'est donc bien que Paris Métropole se contente d'être un cénacle d'élus, car nous n'arriverons à avancer qu'en faisant la preuve de notre aptitude à faire consensus sur des projets partagés.

Je crois que pour que l'enjeu de la gouvernance suscite un vrai intérêt, il faut s'en donner les moyens. Ce n'est pas seulement une question de pédagogie, mais bien de trouver des formes de la démocratie en rapport avec la réalité métropolitaine. J'ai tenté à différentes reprises de renouveler l'exercice. À la Maison de l'Europe, par exemple, dès la fin 2007, nous avons monté des débats publics intitulés « entre villes et métropoles ». Le fonctionnement métropolitain était débattu sous l'angle de la vie quotidienne des habitants (ségrégation scolaire, intégration de la logistique en ville, l'immigration comme richesse et source de créativité...) avec, en ligne de mire, la comparaison avec des métropoles européennes.

L'expérience de débat citoyen conduite avec Anne Hidalgo, "Imaginons Paris, ensemble demain » a fait la preuve de l'intérêt de cette démarche. L'enjeu est bien de sortir la question 
métropolitaine du champ clos des élus, en posant les bases d'une démocratie des usages, ce qui veut dire oser interpeller tous les usagers de Paris (touristes, métropolitains, parisiens) sur l'avenir de la Ville. Spontanément de nombreuses propositions portaient sur la gouvernance et elles ont provoqué des échanges très mûrs.

Je crois donc qu'il en va de notre responsabilité politique de construire ce débat, car poser la question de la gouvernance c'est répondre à une exigence démocratique : aider les habitants à assumer leurs responsabilités, à s'impliquer dans une citoyenneté active.

\section{Q : Y aurait-il un modèle métropolitain dont vous vous sentez proche en termes de valeurs et de projets ?}

Je ne sais pas si on peut parler en termes de modèle métropolitain, mais il est important de savoir, en revanche, autour de quelles exigences on souhaite construire la métropole parisienne.

On ne construit pas une identité métropolitaine en ayant pour seule boussole la volonté d'éviter des émeutes, ni même celle de bâtir des poches d'excellence. Il faut faire métropole pour réconcilier les différents territoires qui la composent. Le mécanisme de segmentation des territoires est dangereux pour ceux qui sont relégués, mais aussi pour ceux qui acceptent de se laisser enfermer dans un entre-soi désiré, parce qu'il est censé les protéger. Il met en cause l'efficacité de l'économie métropolitaine, provoque un scandaleux gâchis collectif de talent et d'énergie, empêche l'émergence d'une identité métropolitaine positive.
Je souhaite une métropole inclusive qui fasse vivre et grandir les formidables potentiels du territoire, qu'une meilleure coordination pourrait faire éclore ou pousser à leur pleine maturité. «Vu de Tokyo ou de New-York, Paris et Clichysous-bois, c'est la même chose » : car si Paris est connue pour sa qualité de vie, elle l'est aussi pour ses émeutes et ses violences urbaines. Si de Tokyo ou de New York, Roissy ou la Défense c'est Paris, de Tokyo ou de New York, la Seine-Saint-Denis, c'est aussi Paris.

Bien sûr qu'il fallait réparer des décennies de relations d'indifférence, voire de mépris, entre Paris et périphérie. C'était le premier enjeu. Mais cela ne suffit pas, il nous faut accélérer pour répondre aux inégalités et savoir offrir à tous ceux qui vivent dans la métropole un projet qui réponde à leurs attentes, en matière de transport, de logement et d'emploi, mais aussi en étant solidaires, et accueillant et tolérant.

Malgré sa puissance, la capacité de cette métropole à participer pleinement à l'accroissement des interactions avec les autres régions métropolitaines internationales, à s'assurer durablement des avantages compétitifs et à savoir attirer la recherche, les activités et les actifs internationaux les plus mobiles les plus créatifs et les plus diplômés est en question. Cette capacité à rayonner doit être renouvelée et doit prendre appui sur le territoire dans lequel elle trouve ancrage : le rayonnement de la métropole ne saurait être « hors sol» si l'on souhaite qu'il soit durable. Plus encore, c'est la possibilité d'associer excellence et solidarité qui fait problème dans notre environnement économique ouvert, incertain, et producteur d'inégalités croissantes. Ce chantier de la solidarité financière 
est indispensable et urgent pour que ce territoire fonctionne mieux et crée de nouvelles richesses au profit de tous.

\section{Q : Que pensez-vous de la diversité voire de la confusion actuelles des points de vue sur la gouvernance métropolitaine?}

Au départ, la question de la gouvernance part d'un constat assez simple. Du point de vue des habitants d'une métropole, les frontières administratives n'ont pas grand sens ; les Métropolitains sont très directement concernés par des décisions de collectivités voisines de celles où ils résident et sur lesquelles ils n'ont pas aucune prise. C'est donc une question de démocratie : il n'est plus concevable que des décisions lourdes de conséquence pour les citoyens soient prises dans des instances qui échappent à leur contrôle.

Aller vers une gouvernance métropolitaine, ce n'est donc rien d'autre que formuler l'exigence qu'un espace, réellement vécu, prenne une forme politique. Je crois que cette idée, inconcevable il y a dix ans, a fait aujourd'hui son chemin.

Si une certaine confusion a pu s'immiscer, c'est sans doute que le débat s'est figé autour d'une fausse opposition. D'un côté, l'on a agité l'idée d'une solution radicale et rapide. C'est ce qu'on retrouve dans la proposition d'une fusion des départements de Paris et de petite couronne, que la Commission Balladur avait portée notamment. Cette manière d'envisager le problème ignore l'Histoire, mais aussi la réalité du fait métropolitain, et notamment son espace réel. Une telle idée, que Bertrand Delanoë a pourtant repoussée, alimente les fantasmes, vite instrumentalisés, d'un Paris hégémonique. De l'autre-je pense à Nicolas Sarkozy - on a repoussé ce sujet de 10 ans, estimant que le projet devait précéder la solution institutionnelle. Renvoyer à plus tard cette question, c'était pourtant accepter une recomposition institutionnelle de l'Île-deFrance qui, aujourd'hui, avance masquée. En procédant de la sorte, on est condamné à prendre acte des rapports de force locaux, et surtout à contourner la question des contradictions naturelles entre les territoires de la métropole.

Pour avancer, je crois au contraire qu'il faut commencer par définir un chemin politique vers la gouvernance de la métropole. Il faut, là-dedans, de la modestie et de la décision. Modestie, car il est illusoire de penser qu'il existe une solution simple (par fusion de je ne sais quel niveau institutionnel) à des questions qui concernent près de 10 millions d'habitants. Décision, car on ne sortira pas du statu quo par des petits ajustements sans rupture. Il faut viser des règles, des dispositifs, des moyens et des stratégies qui permettent de partager l'action publique entre territoires, pour servir la cohérence et l'effectivité de leurs fonctions respectives. Paris Métropole est évidemment le lieu qui doit se saisir de cet enjeu. Je crois que Paris métropole est mûr et qu'il faut lui donner des compétences et le positionner comme l'outil de régulation des débats métropolitains.

Ce pragmatisme résolu, qui prend en compte les expériences des autres métropoles mondiales, est enfin un bon moyen d'éviter la crispation que suscite la question du « bon périmètre ». Il n’y a pas de périmètre idéal pour la métropole. 
En fonction des enjeux, des rapports de force, nous pourrons avancer vers des mécaniques de régulation à géométrie variable. 